\title{
50 A LOCAL REVIEW OF THE NCEPOD AUDIT OF PARENTERAL NUTRITION - WHAT CAUSED THE 'MIXED BAG'?
}

E Murray, V Kalansooriya, R Campbell, M Green, T Beattie, G B Turner. Nutrition Support Team, Royal Victoria Hospital, Belfast, Northern Ireland

\subsection{6/gutjnl-2013-305143.59}

Introduction The report, "A Mixed Bag", published by NCEPOD in 2010 was a review of Parenteral Nutrition (PN) in hospitalised patients. It demonstrated that only $19 \%$ of adults received care deemed to represent good clinical practice. Particular attention was drawn to poor documentation of nutritional issues.

Aims/Background To audit the administration of Parenteral Nutrition within the RVH, Belfast in direct comparison with NCEPOD report and to identify where discrepancies existed.

Method Consecutive patients started on PN at the time of the NCEPOD report were identified using pharmacy records. Each patient's medical, dietetic and nutrition nursing notes were independently reviewed using the NCEPOD PN Questionnaire.

Results 27 patients were identified with patients excluded due to missing casenotes. No treatment goal was documented for $89 \%$ of patients. Twelve percent of patients had PN started at a weekend, refeeding risk was not documented for these patients but was for all others. The initial bag was inappropriate for patient needs in $17 \%$, the majority of these started at the weekend. While combining records revealed good clinical monitoring, this was poor in medical notes compared with dietetic/nursing records. Metabolic complications occurred in $39 \%$, similar to NCEPOD. There was a universal lack of documentation regarding central venous catheter insertion.

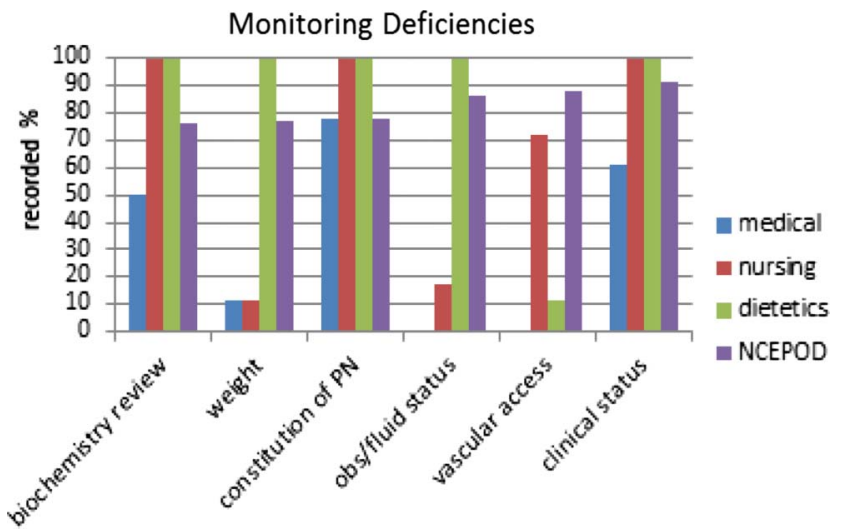

Figure 1 


\section{Abstracts}

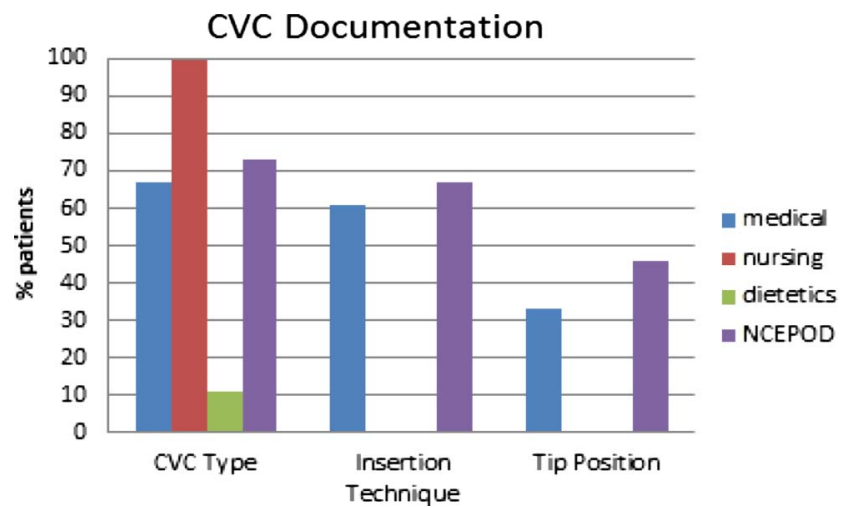

Figure 2

Conclusion Our audit reiterates the findings of the NCEPOD report, with particular regard to documentation and complications. Patients started at the weekend appeared to have poorer documentation. The development of a PN proforma or clinical check list may be useful to identify and reduce risks/complications. 